\title{
NATURAL AND ARTIFICIAL PRODUCTION OF ATMOSPHERIC PRECIPITATION
}

\begin{abstract}
A
DISCUSSION on the "Natural and Artificial Production of Atmospheric Precipitation" was held on August 10 at Edinburgh as part of the programme of Section A (Mathematics and Physics) of the British Association. The session was opened by the president of the Association, H.R.H. the Duke of Edinburgh.
\end{abstract}

B. J. Mason (Imperial College of Science and Technology, London) read the first paper and began by describing the mechanisms responsible for the release of natural precipitation, emphasizing that an understanding of the fundamental physical processes is essential if artificial rain-making experiments are to be carried out intelligently and their results assessed in a realistic manner. He said that the collision and the resultant coalescence of droplets having differential rates of fall, while capable of producing only drizzle or light rain from layer clouds, can release a heavy shower from a deep, vigorous, cumulus cloud by initiating a weter-drop chain reaction. This process will be favoured in tropical regions, particularly in unstable maritime air-masses, where sea-spray may supply the larger droplets required to start the coagulation process. In temperate latitudes and well inland, showers are more likely to be released by the action of ice crystals. Growing initially by diffusion, the crystals afterwards sweep up supercooled cloud droplets to form pellets of soft hail, which may melt to form raindrops. Rain falling from deep layer-clouds generally originates from melting snowflakes, which are formed by the coalescence of ice crystals. In cumulus clouds containing strong updraughts and high concentrations of liquid water, a water-drop chain reaction may be initiated by the shedding of large droplets from hailstones which amass supercooled water at a rate greater than it can be frozen.

In describing and assessing the results of artificial rain-making experiments, which attempt to induce precipitation by 'seeding' suitable clouds with pellets of dry ice, crystals of silver iodide or small waterdroplets, Mr. Mason said that there has been a distinct tendency to draw spectacular conclusions on the basis of too few observations, and that too little attention has been paid to the need for adequate control experiments. However, many cases have been reported of rain falling from clouds a few minutes after seeding. Although in any particular case it is not possible to ascertain that this would not have happened without seeding, in many careful dryice experiments carried out in Australia by the Council for Scientific and Industrial Research (now the Commonwealth Scientific and Industrial Research Organization), under the direction of Dr. E. G. Bowen, rain has been observed to fall from seeded cumulus clouds, while similar clouds in the immediate vicinity produced no precipitation. Since, for a high probability of success in the dry-ice experiments, the cloud top must be colder than $-7^{\circ} \mathrm{C}$. and rain is likely to fall naturally if it is colder than $-12^{\circ} \mathrm{C}$., there is only a narrow range of conditions in which seeding with dry ice can anticipate natural events. The position is still more unfavourable in the case of silver iodide, which suffers the additional disadvantage that it becomes inactive as an ice nucleus when exposed to strong sunlight for about one hour. Calculations indicate that spraying small water-droplets into the base of the cloud should be a more efficient method of releasing showers from warm cumuli, and the results of recent experiments in Australia are encouraging.

The introduction of dry ice into supercooled layer clouds tends to produce 'over-seeding' and therefore to inhibit precipitation. This danger is reduced by the dissemination of silver iodide from the ground; this method is being used extensively in the United States, but an accurate assessment of the results will be possible only after extended trials in many different regions, comparing the precipitation on a statistical basis with that produced naturally in corresponding control areas. Generally layer clouds will produce little precipitation unless they possess considerable depth, in which case their tops may often be sufficiently cold for precipitation to be released naturally.

Mr. Mason said that there is little doubt that precipitation may be released by seeding techniques. The important questions are how much can be so released which would not fall naturally, and whether the time of release and hence its distribution can be altered. It is possible that, by seeding, the onset of precipitation from a growing cumulus may be advanced by a few minutes, and from frontal cloud systems by perhaps a few hours. This might be of importance in increasing the rainfall in a given area; but judging the optimum time for seeding would, in most cases, be very difficult. It seems unlikely that the rainfall pattern over large areas can be altered materially by cloud seeding, although local modifications may be effected.

It was suggested by Mr. Mason that perhaps the greatest value of rain-making experiments is to be found in the impetus they have given to fundamental research in the physics of clouds and precipitation; they should be continued as scientific experiments in close collaboration with theoretical and laboratory work on the fundamental physical problems, for only in this way can their potential economic value be satisfactorily assessed.

F. H. Ludlam (Imperial College of Science and Technology, London) emphasized that the time-scale of precipitation processes is controlled mainly by the macroscopic features of cloud development, and showed by time-lapse photographs of cumulus clouds that the life-history of a particular mass of cloudy air may be completed in a few minutes. He went on to discuss quantitatively the formation of raindrops and hailstones in shower clouds, tracing the growth of these elements by coagulation in relation to the elapsed time and their height above the cloud base. His calculations show that a droplet of sea-spray of radius about $30 \mu$ is capable of releasing a shower via a water-drop chain reaction in a warm cumulus cloud having a strong updraught; alternatively, this process can be started by a wet hailstone in the manner described by Mr. Mason.

It was Mr. Ludlam's opinion that, even in temperate latitudes, rain is released from shower clouds without the aid of ice crystals ; but more critical observations are needed. He also discussed the possibilities of inducing and inhibiting showers by seeding techniques, pointing out that seeding can be used as a tracer in studying the further development of a cloud. For 
this reason, and also because he was more optimistic about their potential economic value than other speakers, he hoped that such experiments would be conducted on an increasing scale in the British Isles.

In a paper entitled "Radár Echoes from Precipitation", R. F. Jones (Meteorological Office) pointed out that normal radar equipments working on wavelengths of 3 or $10 \mathrm{~cm}$. can, at ranges greater than ten miles, detect only that part of the cloud in which there are either raindrops, ice crystals exceeding $1.5 \mathrm{~mm}$. diameter, aggregates of ice crystals or ice crystals with a wet surface. He described some results obtained with the radar equipment at East Hill (near Dunstable) which has both position-plan indicators and range-height indicators to give a three-dimensional picture of the echoes received from precipitating clouds. Different weather types are associated with markedly different radar responses, a fact which was clearly illustrated by photographs of the echoes obtained from cloud systems associated with a warm front, a cold front and an occlusion, all of which were of very different character from those obtained from shower clouds. The echoes from layer clouds are generally of limited vertical extent and contain a narrow band of great intensity (the 'bright band'), whlle those from cumulo-nimbus appear as narrow vertical columns attaining heights of perhaps $40,000 \mathrm{ft}$.

Mr. Jones went on to relate the appearance of the echoes, the rate of ascent of their tops and the temperatures of their summits as measured by aircraft, to the mechanisms of precipitation release. In particular, he associated the appearance of the 'bright band' in layer clouds with the melting of snowflakes, and the echoes from cumulo-nimbus (which show no 'bright band') with the existence of large waterdroplets, often supercooled, which must have formed by the coalescence process. He reported on some aircraft observations which appear to support these interpretations.

In a paper read by $H$. P. Palmer, Dr. A. W. Brewer (Oxford) stated that in Great Britain nearly all rain originates from ice crystals, and because of this it is a reliable rule in weather forecasting that cumulus clouds will not give showers until the temperature of their summits falls below $-10^{\circ} \mathrm{C}$. As first suggested by Bergeron, an ice crystal will grow rapidly in a supercooled water cloud; but it is important to consider the origin of these crystals. Suitable ice-forming nuclei are found in the atmosphere; but they are comparatively rare. Their concentration has been measured by Findeisen et al. in Prague, and also in Oxford, by the cooling of air by expansion in Wilson cloud chambers. In the relatively polluted air of these cities the ice nuclei were present in concentrations of only about 1 per $\mathrm{m}^{3}$ at $-10^{\circ} \mathrm{C}$. Dr. Brewer suggested that ice crystals must multiply during their growth by sublimation since it is not until much lower temperatures than $-10^{\circ} \mathrm{C}$. are reached that the ice-forming nuclei are sufficiently numerous to account for all the raindrops which are produced. $\mathrm{He}$ reported that ice crystals which are growing rapidly and have dendritic structures release very small splinters of ice. Thus, although only a very few ice crystals may appear originally in a supercooled cloud at $-10^{\circ} \mathrm{C}$., they will grow rapidly in a form liable to fracture; several splinters may form at each fracture, and themselves act as ice nuclei. In this way, a sufficient number of ice crystals may be formed to account for the number of raindrops reaching the ground.
I. C. Browne (Cambridge) described his use of a radar set with a vertical beam and range-amplitude display to study precipitating clouds, in conjunction with observations of the mass and electrical charge of raindrops and the electric field at the ground. $\mathrm{He}$ has made a particular study of the variations of echo intensity with height in order to determine the mass and growth-rate of the precipitation elements. In particular, thunderstorms sometimes show a striking increase of echo intensity with decreasing height in a region beneath the freezing-level, which affords some evidence for the growth of drops by coalescence and their multiplication by a chain reaction. The observations yield a value for the updraught velocity in the chain-reaction region. In warm-front rain the echo intensity indicates that at about $1 \mathrm{~km}$. above the melting-level ice crystals are growing at a rate of about $150 \mu \mathrm{gm} . / \mathrm{km}$. Calculation indicates that they could grow at only $50 \mu \mathrm{gm}$. $/ \mathrm{km}$. by sublimation, the inference being that they were growing at about $100 \mu \mathrm{gm} . / \mathrm{km}$. by accretion. The observations also indicate that near the $0^{\circ} \mathrm{C}$. level each snowflake, on the average, coagulates with another.

\section{B. J. MASON}

\section{SEED PATHOLOGY*}

\section{By DR. MARY NOBLE \\ Department of Agriculture for Scotland}

\section{Agricultural and Horticultural Aspects}

CVEN the earliest agriculturists realized the general 1 desirability of using good seed; but it is very surprising that to-day so little attention is paid to the presence of disease in what is otherwise regarded as 'good' seed. I refer in this respect to true seed in the botanical sense, the health of other planting-stock having been greatly improved recently owing to certification schemes covering potatoes, soft fruits, etc. This is probably because most seed-transmitted parasites do not have any immediate effect upon germination; they do not kill the seed outright but multiply upon the emerging seedling, which often then succumbs to the attack; or, as in the case of loose smut of barley, the parasite may pass most of its life-cycle as a symbiont. Such seed, if tested by orthodox methods, is classed as 'good seed' but is, in fact, responsible for considerable loss of crops not only in Great Britain but also the rest of the world. To take, as a case in point, an example from one of our most familiar crops - the turnip. Dry rot of turnip caused by Phoma lingam has been known for very many years; but although the farmer has known this disease as a rot of the root, the growers of swede and turnip seed only recognized it comparatively recently. Swede roots are replanted for seedproduction in the second year, and it was found that the base of the shoot was often attacked by the disease known as 'canker'. The important point is that it was not until 1930 that this 'canker' was proved to be merely 'dry rot' in another form; and what was even more important, that the fungus also infects the seed heads, the pods and even the seed itself but without causing reduction in germination. Until this point was properly understood, turnip and swede seed samples were frequently severely infected by dry rot, because although the seed grower was

* Substance of a paper read on August 13 before Section K (Botany) of the British Association meeting at Edinburgh. 